Unternehmen und biologische Vielfalt

\section{Naturkapital Deutschland}

Ökonomisch betrachtet stellt die Natur ein Kapital dar, aus dem Dividenden in Form von Ökosystemleistungen fließen. Doch obwohl Unternehmen mit Sach- und Humankapital sorgfältig umgehen, übersehen sie oft, dass auch das Naturkapital einen wichtigen Beitrag für die Wertschöpfung darstellt. Von Bernd Hansjürgens und Barbara Wieler

D er Zustand vieler Ökosysteme ist alarmierend. Der Verlust der biologischen Vielfalt (Biodiversität) schreitet voran. Mit rasender Geschwindigkeit sterben heute Arten aus - 100- bis 1000mal schneller als vor dem menschlichen Einfluss. Urwälder werden gerodet, Flüsse verschmutzt, die Meere überfischt und Böden überdüngt und ausgelaugt. Der Klimawandel setzt Korallenriffen so stark zu, dass sie in wenigen Jahrzehnten absterben werden.

Bis zum Ende dieses Jahrhunderts werden rund ein Drittel der heute bekannten Arten, die Vielfalt der Ökosysteme und die genetische Vielfalt eingeschlossen, ausgestorben sein.

\section{Verlust der biologischen Vielfalt und Unternehmen}

Die Ursachen für den Verlust der Biodiversität und die Degradation von Ökosystemen sind:

】 Landnutzungsänderungen,

- Übernutzung / Raubbau,

I Verschmutzung,

- Verbreitung gebietsfremder Arten,

- Klimawandel und seine Folgen (z. B. Wüstenbildung, Erwärmung).

Unternehmen sind hieran in vielerlei Weise beteiligt, denn sie stehen in einer engen Wechselbeziehung mit den Ökosystemen, die sie in Anspruch nehmen. Einerseits sind sie von intakten Ökosystemen und deren Leistungen, den Ökosystemleistungen, abhängig. Für eine Vielzahl von Industrie- und Han- delsunternehmen, für den Primärsektor und die Energiebranche sind natürliche Ressourcen sogar entscheidend für den Unternehmenserfolg. Seien es Rohstoffe, Wasser zum Kühlen und Bewässern oder fruchtbare Böden. Andererseits nehmen die Unternehmen durch Entnahme, Verschmutzung und Landnutzungsänderungen Einfluss auf die Natur.

\section{Unsichtbares sichtbar machen}

Ein Grund für die Verschlechterung des Zustands der Ökosysteme und den Verlust der biologischen Vielfalt ist ihre mangelnde Sichtbarkeit in Entscheidungen. Ökosystemleistungen wie die Filterfunktion von Böden, die Reinigungsleistungen einer Aue oder der Erosionsschutz durch Hecken finden sich nicht oder nur selten in Abwägungen über Investitionen, Ressourcenbeschaffung oder Landnutzung wieder - weder monetär noch in anderer Form. Doch nur das, was wir sehen und wertschätzen, berücksichtigen wir angemessen in unseren Entscheidungen.

Die viel beachtete internationale Studie „The Economics of Ecosystems and Biodiversity“, kurz TEEB, hat es sich daher zur Aufgabe gemacht, den Wert der Natur sichtbar zu machen. Unter dem Titel „Die Unternehmensperspektive: Auf neue Herausforderungen vorbereitet sein" erschien nun eine Broschüre des deutschen Folgevorhabens der internationalen TEEB-Studie. Die Broschüre bezieht sich auf den Kontext, in dem sich
Unternehmen in Deutschland befinden. Sie führt Unternehmen an die Thematik heran und zeigt anhand von Praxisbeispielen und wissenschaftlichen Erkenntnissen, wie Unternehmen und biologische Vielfalt zusammenhängen. Gleichzeitig werden praxisorientierte Lösungsvorschläge präsentiert, Biodiversität in Entscheidungen $\mathrm{zu}$ berücksichtigen.

Es sind vor allem fünf Gründe, warum Unternehmen sich mit dem Thema biologische Vielfalt und Ökosysteme auseinandersetzen sollen. Dies bietet ihnen zugleich die Möglichkeit, Risiken in Chancen umzuwandeln:

I Ressourcensicherung. Der gesicherte Zugang und die kostengünstige und nachhaltige Versorgung mit natürlichen Ressourcen (z. B. Bodenschätze oder Frischwasser zum Kühlen) sind insbesondere für Unternehmen entscheidend, die auf Inputs aus der Natur und entsprechende Vorprodukte angewiesen sind.

- Marktnachfrage und Kundenverhalten. Vermehrt richten sich Kundenwünsche auf Produkte, die umweltbezogen unbedenklich sind. Insbesondere Label-affine und einkommensstarke Konsumenten sind bereit, für ein Mehr an Biodiversität tiefer in die Tasche zu greifen.

- Staatliche Regulierung. Obwohl Unternehmen in Deutschland mit Blick auf den Umwelt- und Ressourcenschutz bereits heute zahlreichen Gesetzen und Vorschriften unterworfen sind, ist das nicht das Ende der Fahnenstange. Aufgrund steigender gesellschaftlicher Anforderungen wird sich die Regulierung auch zukünftig weiter ändern.

- Innovation. Lernen aus der Natur, ihre Prozesse und Mechanismen nachahmen kann für Unternehmen wichtige Impulse für Innovationen geben.

- Unternehmerische Verantwortung. Viele Unternehmen schützen die Natur und die Ökosystemleistungen, weil sie Teil der Gesellschaft sind und Verantwortung tragen wollen. Nicht immer werden diese Vorleistungen jedoch am Markt honoriert. 


\section{Biodiversität in das Management integrieren}

Für Unternehmen gibt es vielfältige Möglichkeiten und Ansatzpunkte, zum Erhalt der biologischen Vielfalt beizutragen. Es kann auf zahlreiche Ansätze und Erfahrungen zurückgegriffen werden:

- Wenn Unternehmen ganz am Anfang stehen, scheinen biologische Vielfalt und Ökosysteme nur schwer greifbar. Dabei werden in bestehenden Managementsystemen wie EMAS oder ISO 14001 bereits einzelne Aspekte adressiert. Wer sich einen ersten Überblick verschaffen möchte, auch über das betriebliche Umweltmanagement hinaus, dem bieten die Checklisten der Business and Biodiversity-Initiative oder der Biodiversitäts-Check für Unternehmen einen guten Einstieg.

- Starken Einfluss auf die Natur nehmen Unternehmen vor allem bei Ausund Neubauten von Abbau- und Produktionsstätten. Hier bieten sogenannte Ökokonten die Möglichkeit, weit im Voraus Ausgleichsflächen zu schaffen, welche im Fall eines unvermeidbaren Eingriffs als Ersatzfläche gemäß Bundesnaturschutzgesetz „verrechnet“ werden können. Unternehmen profitieren von weniger Bürokratie, die Natur von frühzeitigem Handeln.

I Besonders wichtige Handlungsfelder, wie beispielsweise Liegenschaften oder der Einkauf, können mit Hilfe von Leitfäden oder Richtlinien umgesetzt werden. Standards, Zertifikate und Labels zu verschiedenen Nachhaltigkeitsaspekten können hierbei als Orientierung dienen.

- Auch in der Berichterstattung spielt das Thema Biodiversität eine Rolle. So formuliert die weltweit akzeptierte Richtlinie zur Nachhaltigkeitsberichterstattung der Global Reporting Initiative (GRI) bis zu fünf Indikatoren zur biologischen Vielfalt. Mit den Entwicklungen hin zu einer ganzheitlichen Berichterstattung, die Geschäfts- und Nachhaltigkeitsberichte vereinigt, sowie der Weiterentwicklung der GRIRichtlinie werden auch die Ansprüche

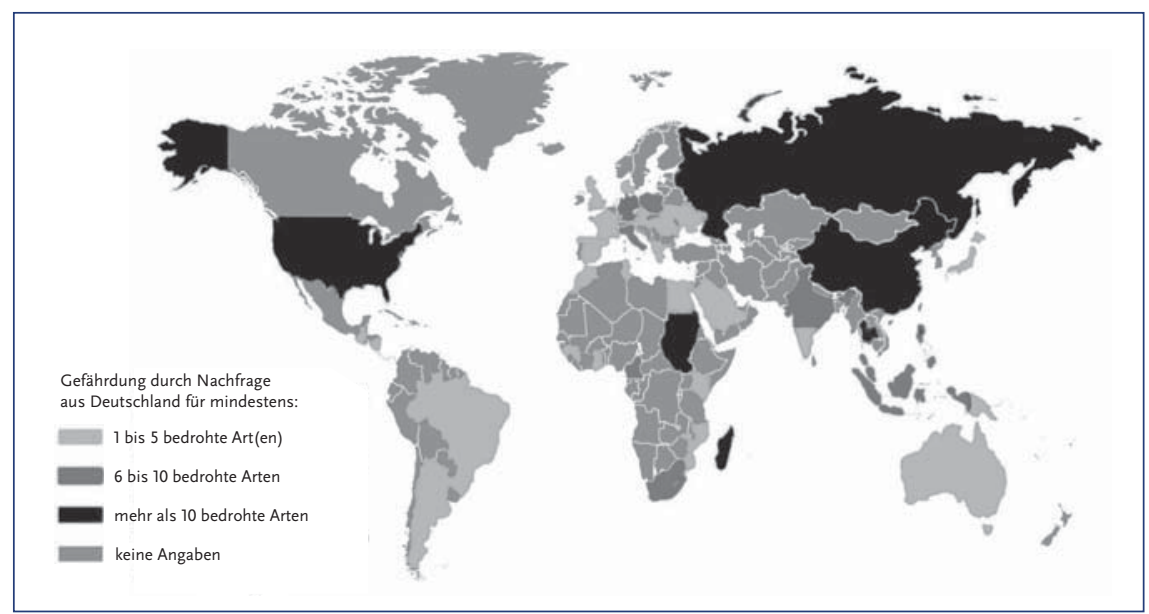

Abbildung 1: Nachfrage in Deutschland setzt bedrohten Arten weltweit zu (Quelle: PwC in Anlehnung an Lenzen et al. 2012)

an die Biodiversitäts-Berichterstattung weiter wachsen.

Noch einen Schritt weiter ging das Unternehmen PUMA, als es vor zwei Jahren seine umweltbezogenen Gewinnund Verlustrechnungen veröffentlichte. Darin wurde deutlich, dass es zahlreiche Ökosystemleistungen in Anspruch genommen hat, für die kein monetärer Wert existiert. Würde man diese Kosten (Externalitäten) einpreisen, müsste das Konzernergebnis um bis zu 145 Millionen Euro korrigiert werden. Besonders interessant ist, dass nur acht Prozent der Wirkungen an den jeweiligen Unternehmensstandorten auftreten, aber 92 Prozent in der Lieferkette liegen.

Dieses Beispiel unterstreicht die große Bedeutung des Lieferketten-Managements. Denn der Einfluss deutscher Unternehmen ist nicht nur hierzulande zu spüren. Über Vorprodukte, deren Anbau oder Gewinnung und Verarbeitung hat Nachfrage in Deutschland einen wesentlichen Einfluss auf Flora und Fauna in den Ursprungsländern. Mithilfe von multiregionalen Input-Output-Modellen konnte ein internationales Team von Wissenschaftlern aufzeigen, wie Nachfrage in Deutschland auf ausgewählte bedrohte Tierarten in den Ursprungsländern unserer Produkte und Vorprodukte wirkt (siehe Abbildung 1).

Doch auch PUMA steht wie viele Unternehmen vor der Herausforderung, das Wissen um ihren Einfluss auf die
Ökosysteme, den Möglichkeiten, diesen zu reduzieren, und unternehmerischen Erfolg in Einklang zu bringen. So sind letztlich alle gesellschaftlichen Akteure gefragt: informierte, sensibilisierte und zahlungsbereite Kunden, verbindliche regulatorische Vorgaben und engagierte und innovative Unternehmen. Es gilt: nur wer auf neue Herausforderungen gut vorbereitet ist, kann auch angemessen mit ihnen umgehen und im Wettbewerb bestehen.

\section{Anmerkung}

Weitere Informationen und Download der Broschüre „Naturkapital Deutschland TEEB DE: Die Unternehmensperspektive: Auf neue Herausforderungen vorbereitet sein“ unter www.naturkapital-teeb.de

\section{AUTOREN + KONTAKT}

Dr. Bernd Hansjürgens ist Professor und Leiter des Departments Ökonomie am Helmholtz Zentrum für Umweltforschung - UFZ und Studienleiter "Naturkapital Deutschland - TEEB DE“.

Prof. Dr. Bernd Hansjürgens, HelmholtzZentrum für Umweltforschung - UFZ, Department Ökonomie, Permoserstraße 15, 04318 Leipzig. Tel.: +49 3412351 233, E-Mail: bernd.hansjuergens@ufz.de

Barbara Wieler ist Senior Managerin bei PricewaterhouseCoopers (PwC) und verantwortet den Bereich Ecosystem \& Climate Change Services.

Barbara Wieler, PricewaterhouseCoopers AG Wirtschaftsprüfungsgesellschaft, Potsdamer Platz 11, 10785 Berlin. Tel.: +49 3026361563

E-Mail: barbara.johanna.wieler@de.pwc.com 
Copyright (C) 2013, IÖW und oekom Verlag. Die Nutzung des Artikels ist Abonnenten von Ökologisches Wirtschaften vorbehalten. Nachdruck und Vervielfältigung des Artikels einschließlich Speicherung und Nutzung auf optischen und elektronischen Datenträgern nur mit Zustimmung der Redaktion von Ökologisches Wirtschaften (http://www.oekologischeswirtschaften.de). 